Supporting Information for

\title{
Colloidal Photonic Assemblies for Colorful Radiative Cooling
}

Hyeon Ho Kim', Eunji Im², and Seungwoo Lee ${ }^{1,2,3^{*}}$

IKU-KIST Graduate School of Converging Science and Technology, Korea University, Seoul 0284I, Republic of Korea 2Department of Biomicrosystem Technology, Korea University, Seoul 0284I, Republic of Korea

${ }^{3} \mathrm{KU}$ Photonics, Korea University, Seoul 0284I, Republic of Korea

*Email: seungwoo@korea.ac.kr

Keywords: Colloids, Opals, Radiative cooling, Photonic Bandgaps

Number of pages: 10

Number of figures: 8

Number of schemes: 0

Number of tables: 0

\section{Table of Contents:}

1. Retrieval of effective refractive index by using s-parameters

2. Numerical calculation of absorptivity/emissivity of opals

3. Self-assembly of opals

4. Microscopic view of the cracks in self-assembled opals

5. Experiments on daytime radiative cooling

6. Calculation of radiative cooling power

7. Additional data for daytime radiative cooling of opals 


\section{Retrieval of effective refractive index by using s-parameters}

Finite-difference, time-domain (FDTD) numerical simulation, supported by 2003-2015 Lumerical Solution was carried out for the theoretical analysis of the effective refractive index of silica opals at the mid-infrared (IR). In particular, we retrieved effective refractive index by using $s$-parameters. ${ }^{[1]}$ The unit cell of opals is shown in Fig. S1a-b. Wavevector (k) of incident light was normal to (111) plane for $s$-parameter calculation. The E-field monitoring in parallel to the incident wavevector was used to set the effective thickness. Because the retrieved effective parameter is intrinsic property, the thickness of the homogeneous slab should be independent on the retrieved parameter. The retrieved effective indices for $200 \mathrm{~nm}, 240 \mathrm{~nm}$, and $290 \mathrm{~nm}$ of silica colloidal opals are shown in Fig. S1c.

(a)

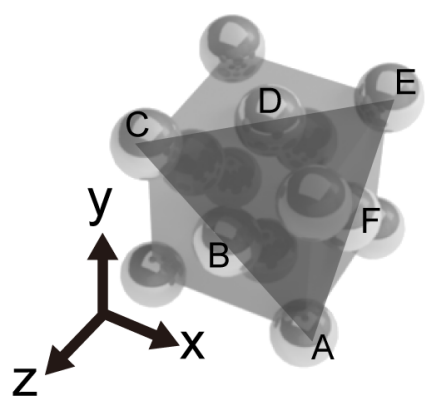

(b)

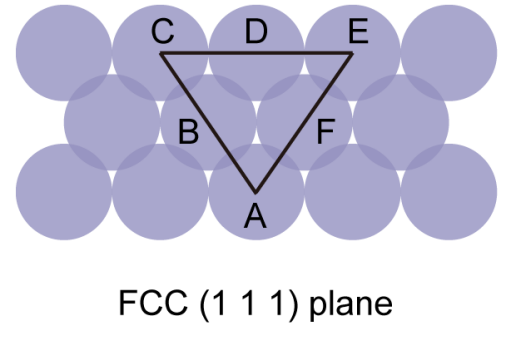

(c)

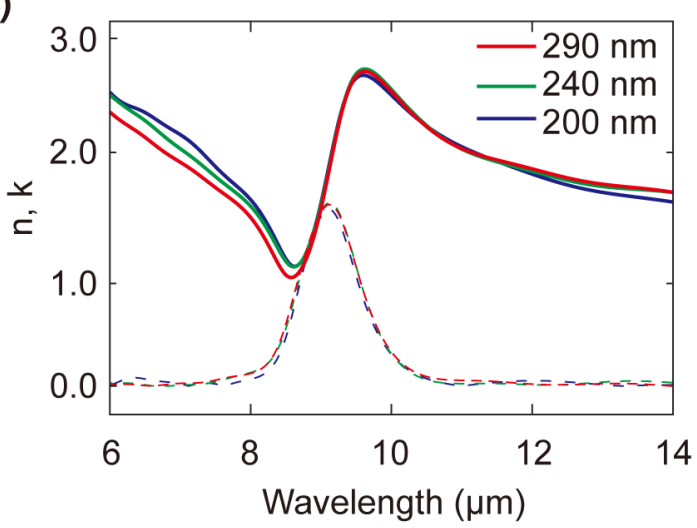

Figure S1. (a) Unit cell of opal with highlight of (111) plane. (b) Schematic for a front view of (111) plane. (c) Mid-infrared (IR) effective refractive index of silica opal composed of differently sized silica nanospheres $(200 \mathrm{~nm}, 240 \mathrm{~nm}$, and $290 \mathrm{~nm})$. Solid and dotted lines indicate real $(n)$ and imaginary $(k)$ refractive indices respectively.

\section{Numerical calculation of absorptivity/emissivity of opals}

Given effective parameters obtained by the $s$-parameter retrieval method, absorptivity/emissivity of the $150 \mu \mathrm{m}$ thick silica opals were numerically calculated by FDTD method at the mid-IR regime (3 $\mu \mathrm{m}$ to $15 \mu \mathrm{m})$. In this regime, opals were approximated by the thin solid film with the effective parameters, presented in Fig. S1 and Fig. 1c. This can be justified by a homogenization theory under the deep-subwavelength scale of opals. From UV to NIR (3 $\mu \mathrm{m}$ wavelength), whole structure of FCC along the same thickness was modeled in this numerical simulation and the absorptivity was numerically obtained. 


\section{Self-assembly of opals}

\section{3-I. Synthesis of silica colloids}

All chemical reagents, including Tetraethyl orthosilicate (Aldrich, $\geq 99.0 \%$ ), ammonia solution (Aldrich, 25\%), and anhydrous ethanol (Aldrich, $\geq 99.5 \%$ ) were used as received. Deionized (DI) water (Milli-Q water) was used as a solvent for the synthesis of silica nanospheres. We synthesized silica nanospheres in a one-step process using the solvent varying method, ${ }^{[2]}$ which is modified the sol-gel Stöber method. ${ }^{[3]}$ Given the mixture of TEOS, ammonia solution, and DI water (respectively 6,8 , and $3 \mathrm{~mL}$ ) we varied the volume of ethanol from 47 to $68 \mathrm{~mL}$. Through this, the size of silica nanospheres were precisely tuned from $200 \mathrm{~nm}$ to $290 \mathrm{~nm}$. The reaction temperature was $60^{\circ} \mathrm{C}$ and the mixture was stirred at a speed of $800 \mathrm{rpm}$ using a magnetic bar for 2 hours. The synthesized silica nanospheres was spun at $300 \mathrm{rcf}$ for $30 \mathrm{~min}$ in a centrifuge and washed with ethanol 5 times. After the ethanol was completely evaporated, the silica particles were dissolved in pure ethanol with a concentration of $10 \mathrm{wt} \%$.

\section{3-2. Assembly of opals}

We assembled opals using gravity sedimentation through solvent evaporation (so called dropcasting). A $3 \mathrm{~cm}$ x $3 \mathrm{~cm}$ crystalline-silicon (c-Si) substrate, which was already washed with isopropyl alcohol (IPA), acetone, ethanol, and DI water in successive way, was coated with polydimethylsiloxane (PDMS) using spin coating (9000 rpm for $60 \mathrm{~s}$ ) and thermally cured in $80^{\circ} \mathrm{C}$ oven for $1 \mathrm{~h}$. Then, $1 \mathrm{~mL}$ of a $10 \mathrm{wt} \%$ silica colloidal solution was drop-casted on the PDMS/c-Si substrate in a $24^{\circ} \mathrm{C}$ oven. During the evaporation of ethanol, silica nanospheres were self-assembled into opaline structures (see Fig. S2).

(a)

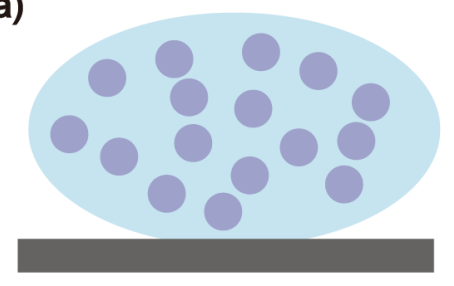

Silica particles spread on substrate (b)

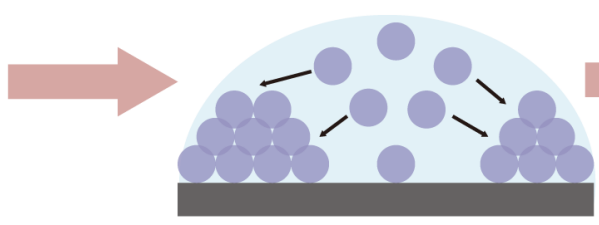

Self assembly into opal (c)

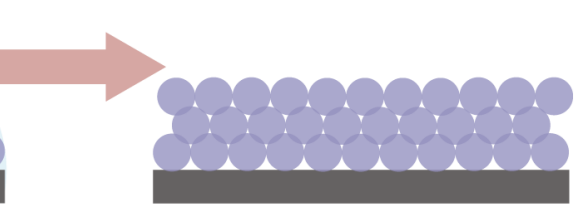

Self assembled silica opal

Figure S2. (a) Drop-casting of silica colloidal solution on the substrate. (b) By gravity, silica nanospheres are self-assembled on the substrate. (c) After the few hours, the solvent was completely evaporated and the assembled silica opals were obtained. 
4. Microscopic view of the cracks in self-assembled opals

(a)

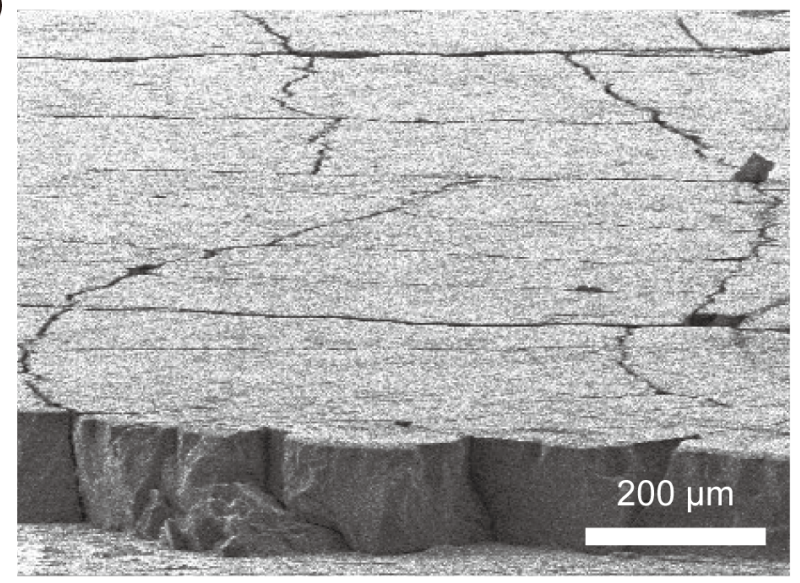

(b)

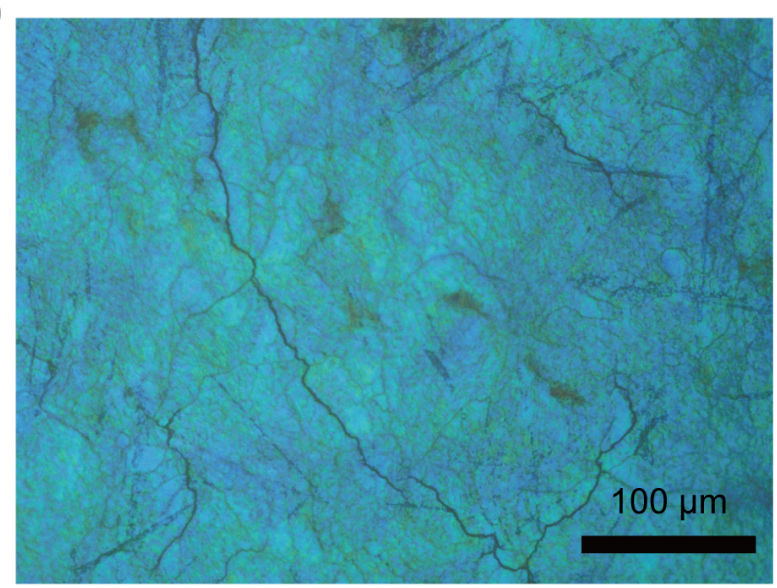

Figure S3. (a) Scanning electron microscope (SEM) and (b) bright field optical microscope (OM) images of the cracks in the self-assembled opals. The intrinsic cracks and point defects are clearly visible in both images, causing an unexpected diffusions and localizations of the incoming sunlight within assembled opals. 


\section{Experiments on radiative cooling}

The measurement of outdoor radiative cooling was carried out in Seongbuk-gu, Seoul with $37^{\circ} 35^{\prime} 24.0^{\prime \prime} \mathrm{N} 127^{\circ} 01^{\prime} 36.4^{\prime \prime E}$ (Korea University campus). Resistance temperature detectors (SA-1 RTD, Omega Korea) were attached to the backside of the samples to measure the temperature and connected to a data logger (OM-CP-OCTRCT, Omega Korea) to record the time-traced temperature variations. The RTD-attached samples were put into the custom-built, thermally isolated box (Fig. S4), consisting of paper box, aluminized polyethylene terephthalate (PET) film, styrofoam, acrylic box, and low-density polyethylene (LDPE) film (see Figure $\mathbf{4 b}$ of main manuscript). As a windshield, the LDPE seals the window. A pyranometer (TES1333R) was placed towards the sky to measure the solar irradiance incident on the sample.

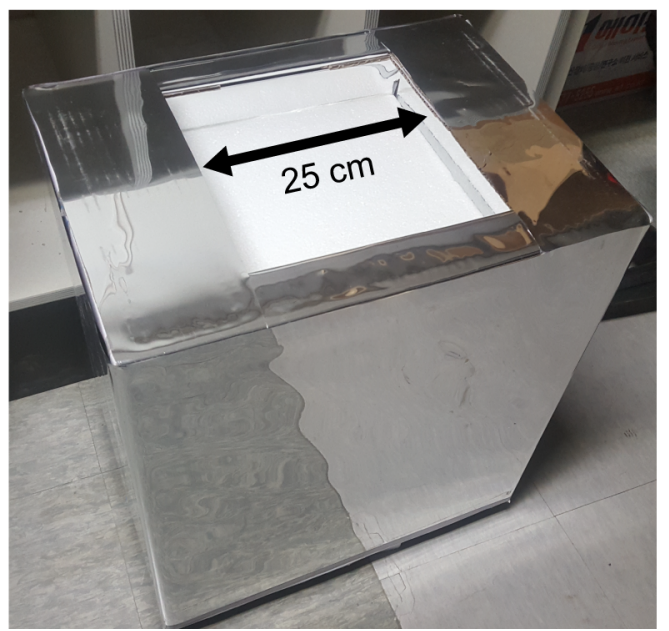

Figure. S4 Outdoor temperature measurement set up without samples. 


\section{Calculation of radiative cooling power}

We calculated the net cooling power as follows: ${ }^{[4]}$

$P_{\text {net }}\left(T_{\text {sample }}\right)=P_{\text {rad }}\left(T_{\text {sample }}\right)-P_{\text {atm }}\left(T_{\text {ambient }}\right)-P_{\text {sun }}-P_{\text {conduction }+ \text { convection }},(\mathrm{S} 1)$

where each term in S1 indicate the total power of the sample, the power of the sample to radiation to the sky, the power absorbed by the sample from the atmosphere, the power absorbed by the sample from the sun, and the power due to the heat exchange with the surrounding environment (from left to right). Each term of the right side is detailed as follows.

$$
\begin{aligned}
& P_{\text {rad }}\left(T_{\text {sample }}\right)=\int_{0}^{2 \pi} \int_{0}^{\pi / 2} \sin (\theta) \cos (\theta) d \theta d \emptyset \int_{0}^{\infty} I_{B B}\left(T_{\text {sample }}, \lambda\right) \epsilon(\lambda, \theta) d \lambda,(\mathrm{S} 2) \\
& P_{\text {atm }}\left(T_{\text {ambient }}\right)=\int_{0}^{2 \pi} \int_{0}^{\pi / 2} \sin (\theta) \cos (\theta) d \theta d \emptyset \int_{0}^{\infty} I_{B B}\left(T_{\text {ambient }}, \lambda\right) \epsilon_{\text {atm }}(\lambda, \theta) \alpha(\lambda, \theta) d \lambda,(\mathrm{S} 3) \\
& P_{\text {sun }}=\int_{0}^{\infty} I_{A M 1.5}(\lambda) \alpha(\lambda, \theta) d \lambda,(\mathrm{S} 4) \\
& P_{\text {conduction }+ \text { convection }}=h_{c}\left(T_{\text {sample }}-T_{\text {ambient }}\right),(\mathrm{S} 5)
\end{aligned}
$$

$\int_{0}^{2 \pi} \int_{0}^{\pi / 2} \sin (\theta) \cos (\theta) d \theta d \emptyset$ is a solid angle within which the sample absorbs or emits heat. $\epsilon, \alpha, h$, $k_{B}, c, \lambda$, and $I_{B B}(T, \lambda)$ indicate respectively emissivity, absorptivity, Planck's constant, Boltzmann constant, speed of light, the wavelength of the radiation wave, and blackbody radiation (i.e., the amount of emitted energy per unit wavelength at different wavelengths and temperature). Note that absorptivity should be equal to emissivity according to Kirchhoff's law. The emissivity in the equation S3 was calculated as $\epsilon_{\text {atm }}(\lambda, \theta)=1-t(\lambda)^{1 / \cos (\theta)}$, where $t(\lambda)$ is the terrestrial transparency window in the zenith direction. In equation $\mathbf{S 4}, I_{A M 1.5}(\lambda)$ is the AM1.5 spectrum of the sunlight. Solar spectrum and terrestrial transparency window appeared in Fig. 1d of main manuscript.

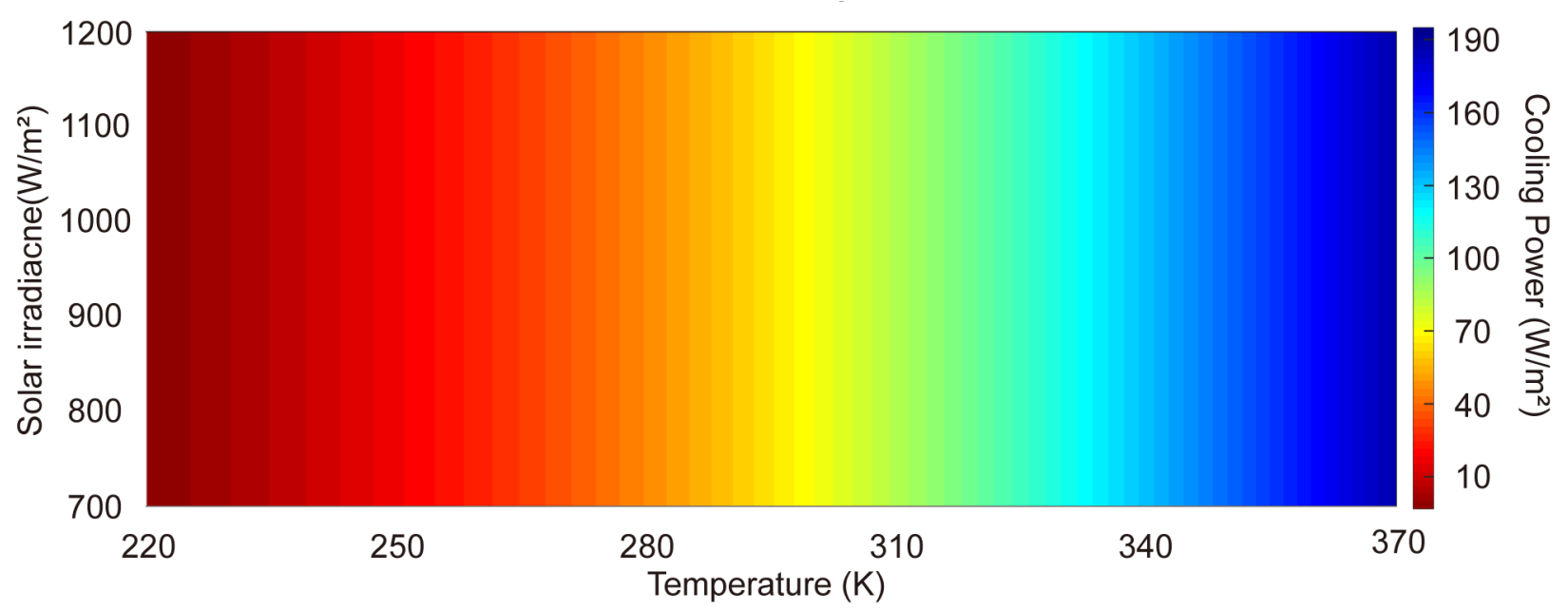

Figure S5. The calculated net cooling power of reddish opals according to the device temperature and solar irradiance. 
7. Additional data for daytime radiative cooling of opals

(a)

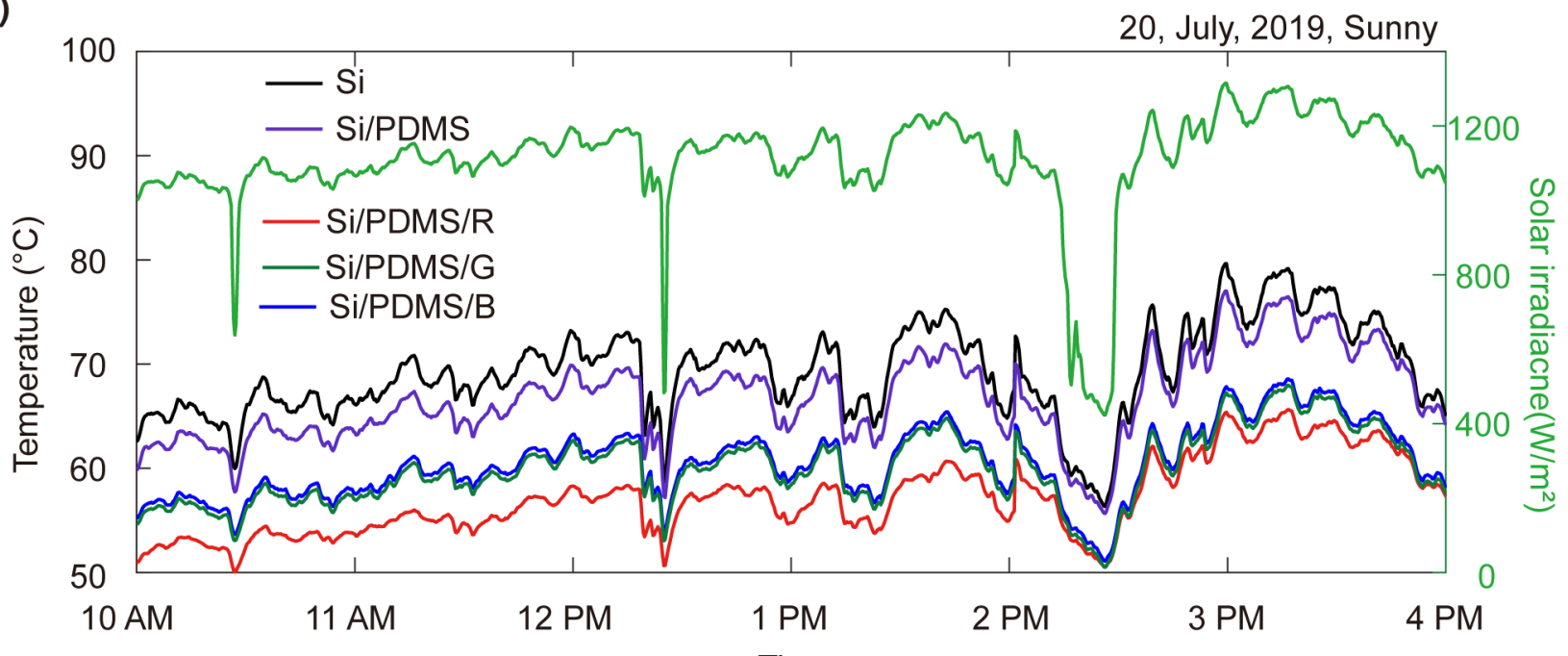

(b)

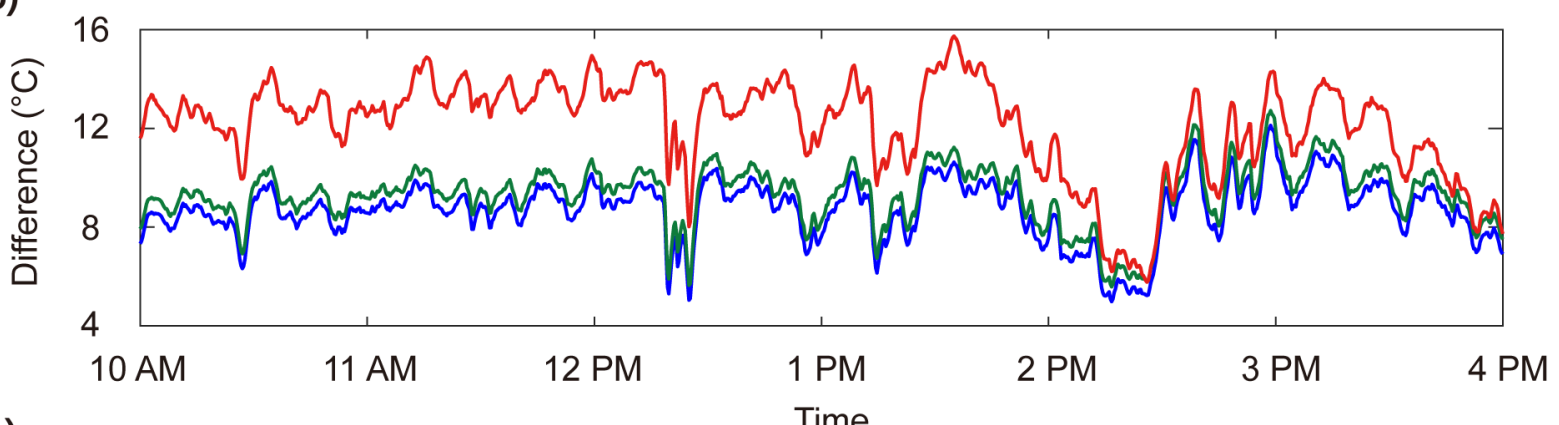

(c)

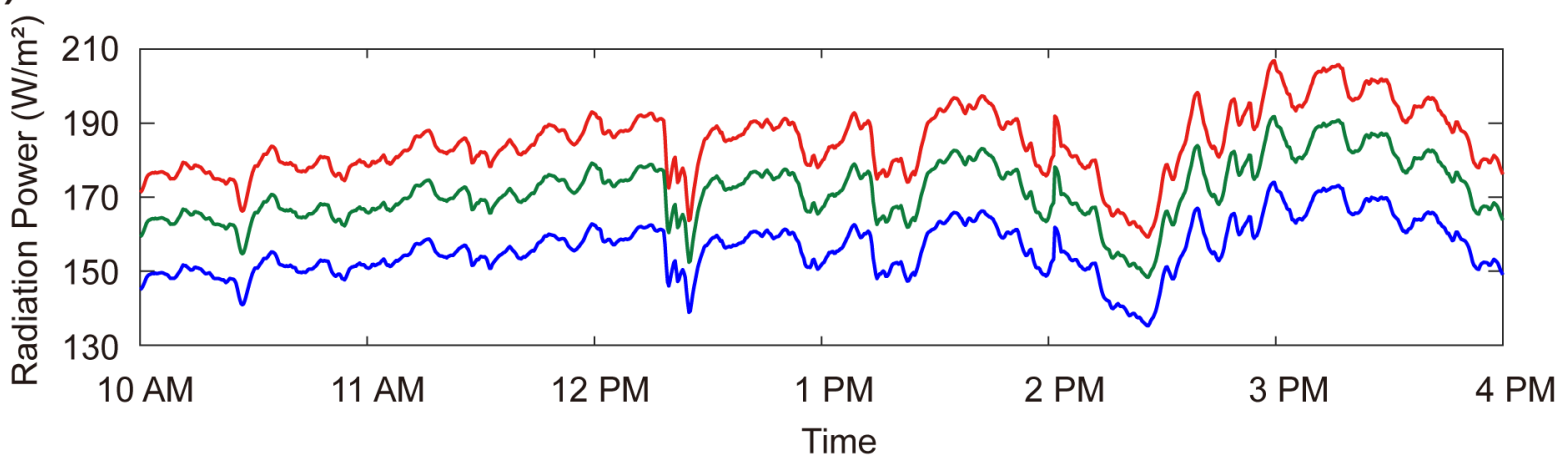

Figure S6. Additional time-traced temperature measurement for outdoor daytime radiative cooling experiment. These experiments were performed during sunny daytime on July $20^{\text {th }}, 2019$. In that day, it was a quite sunny; but, cloud was temporarily passed over the sky. (a) Time-trace temperature variation. (b) Time-trance temperature difference between bare substrate and opals/substrate stacks. (c) Corresponding radiation power. 


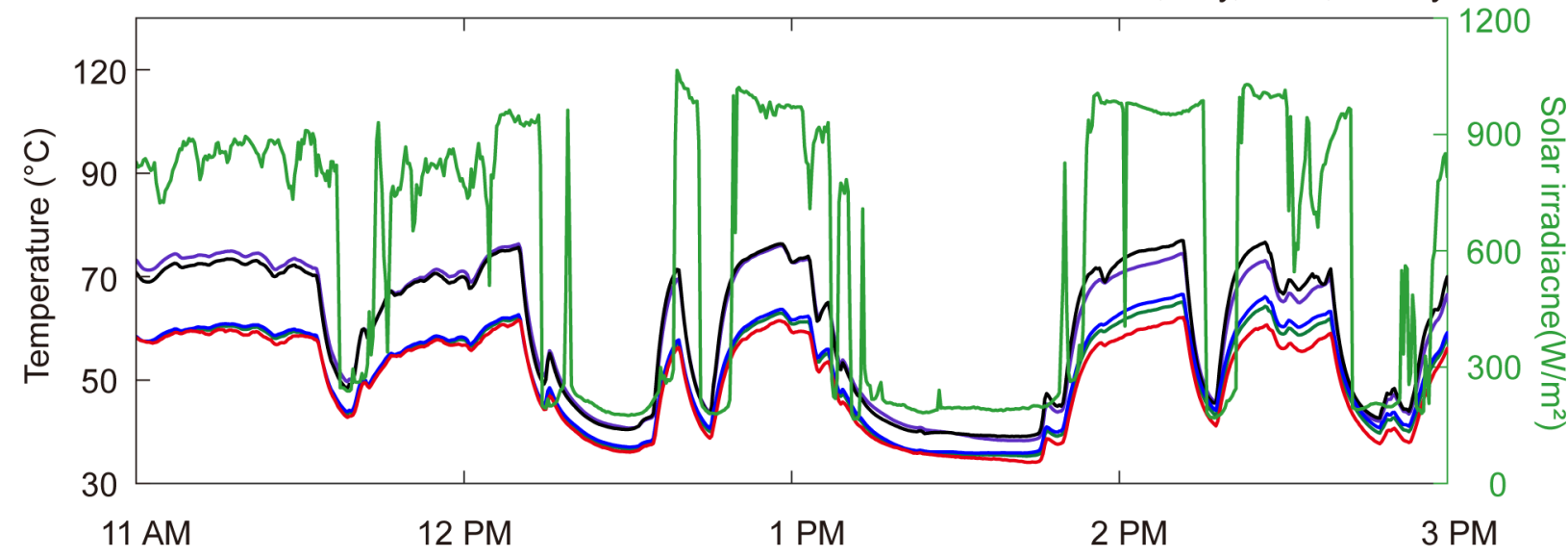

(b)

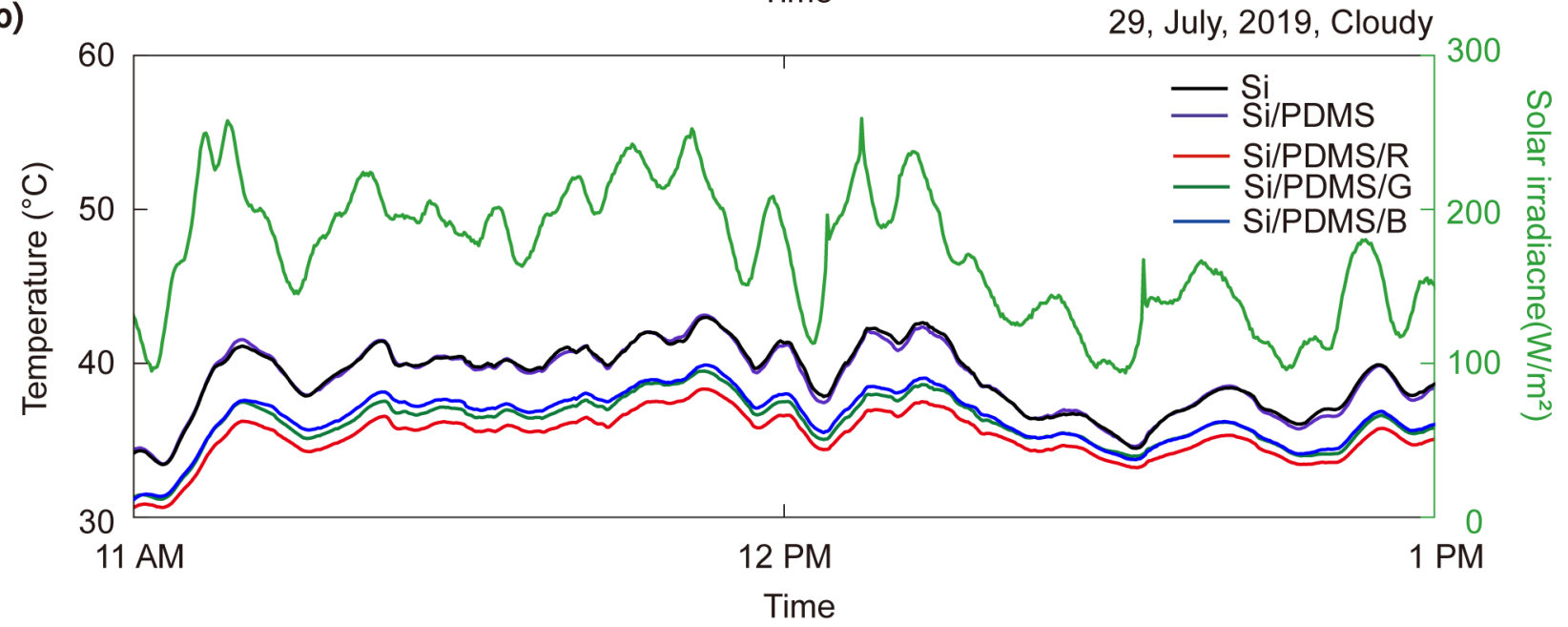

(c)

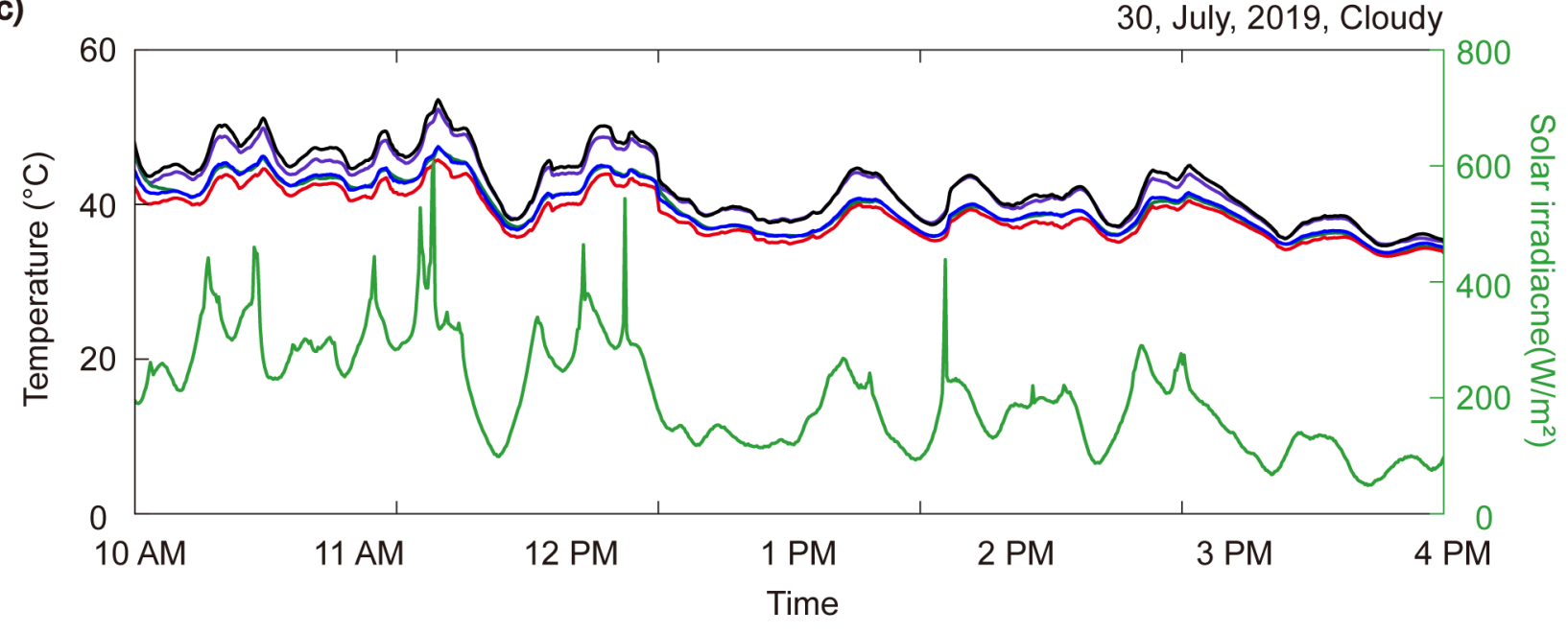

Figure S7. Additional time-traced temperature measurement for outdoor daytime radiative cooling experiment. These experiments were performed during cloudy daytime on (a) July $12^{\text {th }}$, (b) $29^{\text {th }}$, and (c) $30^{\text {th }}, 2019$. 


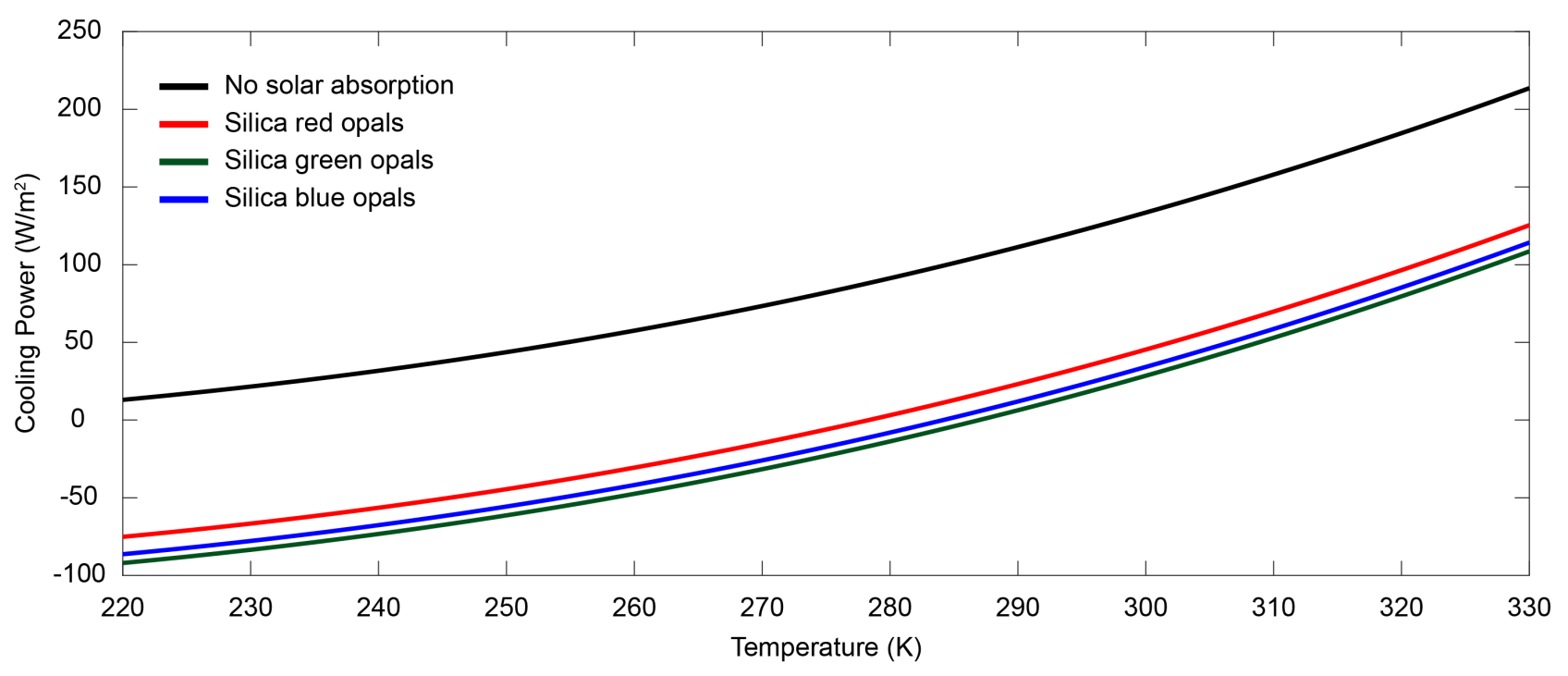

Figure S8. Theoretical comparison of the achievable cooling power between the self-assembled opals and ideal reflector-based radiative cooler (no solar absorption). Here, a flat silver mirror with a $100 \%$ reflection was used as an ideal reflector; silica with same volume compared with opals was assumed to be located at the front of a flat silver mirror. The experimentally obtained parameters for the selfassembled opals (i.e., absorptivity) were used for this calculation. 


\section{References}

1. Smith, D. R.; Vier, D. C.; Koschny, T.; Soukoulis, C. M.; Electromagnetic parameter retrieval from inhomogeneous metamaterials. Phys. Rev. E 2005, 71, 036617.

2. Gao, W.; Rigout, M.; Owens, H.; Self-assembly of silica colloidal crystal thin films with tuneable structural colours over a wide visible spectrum. Appl. Surf. Sci. 2016, 380, 12-15.

3. Stöber, W.; Fink, A.; Controlled growth of monodisperse silica spheres in the micron size range. J. Colloid Interface Sci. 1968, 26, 62-69.

4. Raman, A. P.; Anoma, M. A.; Zhu, L,; Rephaeli, E.; Fan, S. Passive radiative cooling below ambient air temperature under direct sunlight. Nature 2014, 515, 540-544. 